https://doi.org/10.19195/0137-1169.39.1

EDYTA BŁACHUT

ORCID: 0000-0001-9234-465X

Uniwersytet Wrocławski, Polen

\title{
Emotionalisierung des Sprechens durch Metaphorik, Beispiel: metaphorische Ausdrücke mit dem Wort Ventil
}

\section{Vorbemerkungen}

Im wissenschaftlichen Diskurs der letzten Jahre wird das Verhältnis von Sprechen, Sprache und Emotionen immer wiederkehrend und wieder vermehrt zum Thema. So dürfen die bisher angestellten Überlegungen der Forscher über diesen Zusammenhang nicht unerwähnt bleiben. ${ }^{1}$ Ich greife sie auf, indem ich dem theoretischen Teil dieses Beitrags folgende Aspekte des hier behandelten Themas zugrunde lege: das Verhältnis von Sprache und Emotionen (1.1.) und metaphorisches Emotionspotenzial (1.2.). Dann werde ich das Ziel der Analyse klarstellen (1.3.).

\subsection{Zum Verhältnis Sprache - Emotionen}

Aus der heute kaum noch übersehbaren Anzahl von Veröffentlichungen zur Sprache-und-Emotion-Thematik ergibt sich eine großräumige bzw. breit angelegte

\footnotetext{
${ }^{1}$ Der vorliegende Beitrag ist die Ausarbeitung eines nicht publizierten Vortrags, den ich auf der Tagung „Emotionen in Sprache und Sprechen“, die vom 26. bis 28. September 2019 in Wrocław stattfand, gehalten habe. Der Schwerpunkt der Tagung ging thematisch auf wissenschaftlich bereits verbreitete Diskussion um das Verhältnis von Emotionen und Sprache zurück. Im theoretischen Teil basiert mein Beitrag auf ausgewählten älteren wie neueren Publikationen im deutschsprachigen Raum, u. a. Ludwig (1974), Freitag (1975), Fiehler (1990, 2002), Fries (2000, 2004), Schwarz-Friesel (2007), Ortner (2014) und der Sammelband zu „Emotionale(n) Grenzgänge(n) [...]“ hrsg. von Ebert/Gruber/ Meisnitzer/Rettinger (2011) (zu diesen und weiteren Angaben s. Literaturverzeichnis).
} 
Erkenntnis (die ich übrigens in meine Analyse der Beispiele mit einbeziehe), dass Sprache als kommunikatives Mittel auch dazu genutzt wird, subjektive emotionale Zustände (zunächst ohne nähere Unterscheidung zwischen Emotionen, Gefühlen und Empfindungen) mitzuteilen (vgl. z. B. Fiehler 1990, Schwarz-Friesel 2007 oder Ortner 2014). In einer ersten Annäherung an das Thema konzentriert sich die linguistische Forschung a) auf lexikalisch-semantische Kernfragen und b) auf einige pragmatische und funktionale Kernfragen. Durch pragmatisch und kommunikativ orientierte Methoden ergeben sich Emotionen ${ }^{2}$ als Phänomene, die das Sprechen begleiten oder beeinflussen. Empirische Untersuchungen der Texte und Textsorten, orientiert an konkreten Kommunikationssituationen, situieren Emotionsmanifestationen als Ausdruck der individuellen Veranlagung der Sprecher und/oder als Teil interpersoneller Interaktion, die bestimmten soziopsychologischen Regeln unterliegen (s. z. B. Fiehler 2002, Cirko 2019). Im Rahmen der semantisch-lexikalischen Forschungsprojekte dominieren Untersuchungen zum Emotionsvokabular einer Sprache (z. B. Hermanns 2002, Ortner 2014 im Deutschen). Das Verhältnis von Sprache und Emotionen wurde mittlerweile auf der semantisch-konzeptuellen und sozialen bzw. kulturspezifischen Ebene intensiv thematisiert und problematisiert - insbesondere hat der kognitive Ansatz den $\mathrm{Zu}$ sammenhang von Sprache und Emotion erst recht bekräftigt. Nicht zuletzt wurde dieses Thema bei der Diskussion um den Metapherngebrauch im Alltag wie auch im fachsprachlichen Diskurs behandelt. Ausschlaggebend ist hierfür das Metaphernkonzept von Lakoff/Johnson (1980) und Kövecses (1998), wonach sich der Mensch mittels Metaphern ständig neu in Beziehung zur Realität setzt ${ }^{3}$, wobei spezifische Rahmenbedingungen kulturell vorgegeben werden. In Bezug auf diese Rahmenbedingungen heißt es bei den Autoren (z. B. Lakoff/Turner 1989:214): „To study metaphor is to be confronted with hidden aspects of one's own mind and one's own culture". ${ }^{4}$ Viele Fragen im Bereich Metaphern, Kognition und Emotion, basierend auf diesem Metaphernkonzept, haben z. B. Skirl/SchwarzFriesel 2007 aufgegriffen. Abschließend seien kognitionswissenschaftliche und psycholinguistische Ansätze erwähnt, inklusive Konzepte biologisch-physiologischer Art (Verhältnis: Kognition, Gehirn und Emotion), die von linguistischer Seite her unter anderem von Fries 2000 (und später) aufgegriffen werden. In Be-

${ }^{2}$ In diesem Beitrag werde ich die Begriffe Emotion und Gefühl als Synonyme verwenden. Dazu, wie die beiden Synonyme auf dem Gebiet der Sprache-und-Emotion-Forschung und in der emotionspsychologischen Fachliteratur behandelt werden, s. Błachut (2014:129-132).

3 Metaphern entstehen nach diesem Ansatz durch unsere Interaktion mit der Welt und auf diese Weise bildet sich unser konzeptuelles System. Der Mechanismus, mit dem ein abstraktes Konzept in Begriffen des Konkreten konzipiert wird, erzeugt konzeptuelle Metapher. Ihr Wesen besteht darin, „dass wir durch sie eine Sache oder einen Vorgang in Begriffen einer anderen Sache bzw. eines anderen Vorgangs verstehen und erfahren können“" (Lakoff/Johnson 2004:13).

${ }^{4}$ Der Kern ihrer Theorie (Lakoff, Johnson, Turner, Kövecses) besteht darin, dass Metaphern sich auch als Produkte von Metaphorisierungsprozessen begreifen lassen, auf die sich die Kulturmitglieder eingelassen haben und mittels derer sie ihre Realität als sinnvoll erfahren können. 
zug auf die komplexe Sprachproduktion und Sprachverstehen ist die emotionale Komponente nach diesen Ansätzen als Einflussgröße zu betrachten, die kognitive Sprachverarbeitungsprozesse steuert.

Für meine im Titel angekündigte Diskussion benötige ich einen integrativen Ansatz, der die sprachliche, emotionale und kognitive Komponente aufeinander bezieht. So im Detail: Im empirischen Beitragsteil konzentriere ich mich auf schriftlich fixierte Emotionsmanifestationen, die ich anhand exemplarischer Fallbeispiele für die Emotionen wie Wut, Irritation und Frustration (gelegentlich auch andere, von ihnen abgeleitete Emotionen) diskutieren möchte. Mit Emotionsmanifestationen meine ich Formulierungen, in denen Emotionen als einen Sachverhalt begleitende Erscheinungen entweder vom Autor des Textes (dem Journalisten als Textproduzenten) explizit genannt werden oder von den Rezipienten aus dem Formulierten abgeleitet werden können. Im Zentrum dieser Formulierungen steht jeweils eine Ventil-Metapher als sprachliche Kodierung dessen, was der Verfasser meint und empfindet. In dieser Herangehensweise wird vorwegnehmend deutlich, dass auch der Inhalt der jeweils unmittelbar vorausgehenden Textelemente, nicht nur die Form der metaphorischen Äußerungen mitberücksichtigt wird. Wo die Emotionen nicht namentlich genannt werden, lässt sich nur vorsichtig nachvollziehen, welche Gefühle der Verfasser in seinem Artikel als Ventil-Metapher kodiert. So kann die Diskussion besonders hier nur einen deduktiv-interpretativen Charakter haben.

\subsection{Metaphorik und Emotionen}

Für die Zwecke der Diskussion der Beispiele wähle ich aus einer Vielzahl der Theorieentwürfe zur Metaphorik die Einsicht, dass die metaphorische Ausdrucksweise einen konzeptuellen Charakter hat. ${ }^{5}$ Nach dem Ansatz von Lakoff, Johnson, Turner und Kövecses (aus den 1980er Jahren, hier vgl. 1.1.) bedeutet dies, dass in den zu analysierenden Gegenstandsbereich auch die kulturell und/oder intersubjektiv basierten Annahmen mit einbezogen werden, auf deren Hintergrund die Metaphern entstehen, funktionieren und verständlich werden; das eigentlich Dynamische an diesem Prozess rechtfertigt die Vorstellung einer „Tätigkeit“. Das Wesen der Metapher besteht darin, dass konkrete, alltägliche Erfahrungen der Sprecher (ich nenne sie auch Selbstverständlichkeiten des Alltags) auf eine spezifische Prototypik (oder: prototypische kulturelle Erfahrungen) hin zusammengefasst werden. Stark verkürzt lässt sich die metaphorische Tätigkeit als das Übertragen spezifischer Merkmale von einem Gegenstandsbereich zu einem anderen

\footnotetext{
${ }^{5}$ Damit lasse ich die ursprünglichen Beschreibungs- bzw. Erklärungsmodelle unberücksichtigt, die Metapher als verkürzte Vergleiche (Vergleichstheorie), Anomalie oder Regelverstoß (Substitutionstheorie) auffassten. Zur Übersicht über verschiedene Beschreibungsmodelle der Metapher s. Frieling (1996:23ff.), Elsen (2014:207ff.), Abdulrahman (2018:39ff.).
} 
erklären, was es den Sprechern erlaubt, einen Gegenstand aus der Perspektive eines anderen zu sehen. ${ }^{6}$ Dieses „Sehen-als“ (ich verstehe es als einen Prozess der spezifischen Sichtweise, als einen Erkenntnisprozess) wird durch die Metapher als Ausdrucksvariante der Sprache kodiert, die eine übertragene, nicht wortwörtlich gemeinte Bedeutung hat (so z. B. in Skirl/Schwarz-Friesel 2007). Über den so gefassten Metaphernbegriff kann man zur Feststellung kommen, dass Metaphern als alltägliche Formen der Konzeptualisierung immer auch Einstellungen sind, auf deren Grundlage Gefühle, Emotionen, Empfindungen und Erlebenswelt der Sprecher konzeptuell greifbar gemacht werden, und selbst wieder metaphorisch benannt bzw. wiedergegeben werden ${ }^{7}$ (u. a. Kövecses 1990, 1999 beschreibt Emotionsmetaphern ausführlich).

Die Metaphernforscher sind sich darüber einig, dass die Kommunikation durch Metaphern immer durch vertraute, kollektive metaphorische Konzepte erfolgt, die in einer Sprachgemeinschaft geschöpft und verwendet werden. Ausschlaggebend dabei ist, was mit diesen assoziiert oder impliziert werden kann (vgl. z. B. Pielenz 1993:99, Kirchhoff 2015). In Bezug auf Emotionsthematisierung und Emotionsausdruck durch Metaphern ist zu beobachten, dass Metaphern einerseits Emotionen kodieren, andererseits Emotionen auslösen oder transportieren. Der sprachliche Mechanismus von Metaphern lässt sich dann darauf zurückzuführen, dass der emotive Bedeutungsanteil eines Wortes von seiner denotativen Bedeutung getrennt werden muss, um emotive Effekte zu erzielen. Der aufmerksame Sprecher würde vielleicht eine Beobachtung teilen, dass die Theater-bzw. Zirkus-Metaphorik im öffentlichen Diskurs häufig dazu benutzt wird, um die sich verstärkende Theatralisierung von Politik zu kritisieren und den Gefühlen wie Ärger, Unzufriedenheit, Abneigung - dies metaphorisch zu formulieren - Luft zu machen. Die Spiel-Metaphorik stellt - da sich die Gegner in einem Spiel oder Wettkampf nicht immer an die Regeln halten - das unethische Verhalten bloß; sie nimmt auch über die Assoziierung mit einem unschuldigen Kinderspiel den Handlungen der Erwachsenen Ernsthaftigkeit weg. Tiermetaphern werden verschiedentlich benutzt: einerseits um Kritik an Menschen, ja an Gesellschaft zu üben, andererseits um jemanden oder etwas positiv zu bewerten, zu loben. Den diskursanalytischen Studien (z. B. Ortner 2014, Kirchhoff 2015, Abdulrahman 2018) ist in weitestgehender Übereinstimmung zu entnehmen, dass mit dem Gebrauch von Metaphern verschiedene sozial-emotionale Auffälligkeiten kommuniziert werden.

\footnotetext{
${ }^{6}$ Ohne sich hier zu tief in die Auseinandersetzung zu begeben, sei auf einen Überblick über die Metapherntheorien in Frieling (1996) und Abdulrahman (2018) und kognitive Aspekte der Metaphorik in Kövecses (1998), Schwarz-Friesel (2007) zu verweisen.

7 Die Darstellung der Metaphernkonzepte geschieht in der kognitiven Metapherntheorie in der Form „X IST Y” (z. B. GELD IST WASSER oder Geld IST FLÜSSIGKEIT), ähnlich werden Emotionen wiedergegeben, z. B. Emotionen SIND MeER oder Emotionen SIND FlüsSIGKeITEN IN EINEM BehäLTER; LIEBE IST KRANKHEIT.
} 
Metaphern offenbaren die Stellungnahme der Sprecher einem Ereignis oder einer Person gegenüber, d. h. sie verkörpern Gefühle, Eindrücke und Gedanken der Sprecher/der Schreiber zum vermittelten Inhalt.

Im Hinblick auf die Metaphern im Korpus dieses Beitrags betone ich ihr emotionales Potenzial, allerdings ohne der grundlegenden Sichtweise der emotiven Metapherntheorie (z. B. von Bowers/Metts/Duncanson 1985) unkritisch zu folgen, der emotive Inhalt der Metapher sei der einzige Grund, der die Metapher für die Sprecher so interessant mache. ${ }^{8}$ Nicht alle Metaphern sind automatisch und im Voraus emotiv. Ich gehe davon aus, dass das in den metaphorischen VentilAusdrücken vorhandene bzw. aktivierte emotionale Gebrauchspotential für den Zweck gebraucht wird, neben oder anstatt der emotional neutralen Verwendung eines sprachlichen Ausdrucks ein „Mehr“ zu vermitteln, etwas, was sonst schwer $\mathrm{zu}$ formulieren ist, in komprimierter, verständlicher und außerdem lebendiger Form darzustellen und schließlich auch dafür, um die Einstellungen und Gefühle anderer Sprecher/Leser effektiv zu beeinflussen. Durch die Bezeichnung „Emotionalisierung“ im Beitragstitel wird dies abgedeckt: Emotionen motivieren zu bestimmten Äußerungen, aber auch sprachliches Verhalten (Formulieren) kann Emotionen auslösen.

\subsection{Zielsetzung der Analyse}

Wenn der Zusammenhang von Sprache, Emotion und Metapher in den oben skizzierten Vorbemerkungen annährend richtig erfasst wurde, dann muss es methodisch, von der sprachlichen Ebene ausgehend, möglich und begründet sein, diesen sprachlichen Verwendungszusammenhang zu diskutieren. Meine Verfahrensweise war die folgende: das Sammeln von Beispielen mit dem Substantiv Ventil, die Auflistung der Beispiele und ihre Systematisierung nach Form. Emotionen sind interne und subjektive Phänomene, und als solche gehören sie unter anderem in den Forschungsbereich der Psychologie. Sie sind nicht „,in Sprache“ (wie häufig falsch formuliert, s. Fn. 1), aber es ist möglich, über sie zu sprechen. In Bezug auf die geschriebenen Texte kann das Verhältnis wie folgt formuliert werden: Emotionskonzepte werden in Texten kodiert, beim Lesen werden sie dekodiert. Metaphorische Konstruktionen sind sprachliche Ausdrücke, mit denen die Verfasser auf emotionale Zustände und emotionale Prozesse referieren und mittels derer sie die Emotionen beim Leser beeinflussen oder hervorrufen können. Meine Aufgabe sehe ich darin, die gesammelten Metaphern zum Konzept Ventil zu sichten, d. h. sie sprachlich zu charakterisieren, ihre Funktion in gegebenen Textabschnitten zu bestimmen, den emotionalen Hintergrund für ihren Gebrauch zu rekonstruieren.

${ }^{8}$ Vgl. z. B. die Polemik von Kittay (1987) zu der emotiven Metapherntheorie. 


\section{Analyse der Beispiele}

Belege mit der Ventil-Metapher zitiere ich aus verschiedenen journalistischen Texten, die weder thematisch noch chronologisch zusammenhängen. Es sind meistens meinungsbetonte Texte, $u$. a. politische Pressekommentare, aber auch Interviews oder wissenschaftliche Berichte. Die Zitierquelle wird bei den Beispielen in Klammern entsprechend markiert. Im ersten Schritt erkläre ich den Ursprungsbereich von Ventil anhand der sog. ,Domänen-These“ der kognitiven Metapherntheorie, die ich u. a. nach Lakoff/Johnson (1980 und später) formulieren werde (2.1.). Im nächsten Schritt bespreche ich die gesammelten Ventil-Metaphern nach der syntaktischen Verknüpfung metaphorischer und nicht metaphorischer sprachlicher Elemente in jeweiligen Textpassagen und nach den implizierten metaphorischen Bedeutungen (2.2.). Meine Analyse wird einen beschreibendinterpretativen Charakter haben.

\subsection{Vom Lexem zur Metapher}

Die Domänen-These besagt, dass Zusammenhänge zwischen dem menschlichen Begriffssystem und der Strukturierung von Metaphern auf der Grundlage menschlicher Erfahrungen herzuleiten sind. Eine Domäne wird durch Rückgriff auf eine andere (Erfahrungs-)Domäne kognitiv verfügbar gemacht. Bei Lakoff/Johnson

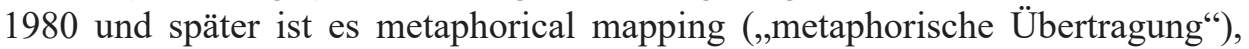
metaphorical projection (,metaphorische Projektion“).

Das Lexem das Ventil (Pl. die Ventile) kommt in zwei Fachbereichen vor - in der Technik und in der Musik. In Wörterbüchern werden die folgenden Bedeutungen verzeichnet: (Technik) Vorrichtung, mit der das Ein-, Aus- und Durchlassen von Flüssigkeiten oder Gasen mechanisch oder von Hand gesteuert wird und (Musik) a) mechanische Vorrichtung an Blechblasinstrumenten, die das Erzeugen aller Töne der chromatischen Tonleiter ermöglicht und b) Mechanismus an der Orgel, durch den die Zufuhr des Luftstroms reguliert wird (z. B. nach Duden. Deutsches Universalwörterbuch 2011). Auf der Grundlage dieser Bedeutungen entstehen in der Alltagssprache typische Attribuierungen: offenes oder geschlossenes Ventil, automatisches oder mechanisches Ventil, defektes, einstellbares Ventil und unzählbare Komposita (z. B. Sicherheitsventil, Steuerventil, Auslassventil, Reduzierventil; Ventilklappe, Ventilschlauch, Ventilsteuerung und viele andere); man kann hier auch einige Derivate auflisten, wie Ventilation, Ventilator, Ventilierung, ventilieren.

Ich folge der Bestimmung, dass Metaphern alltägliche Formen der Konzeptualisierung sind, die in den Erfahrungen der Menschen verankert sind. ${ }^{9}$ Die alltäglichen

9 Diese Grundannahme ist vertreten u. a. in den Arbeiten von Lakoff/Johnson (1980 und später), Lakoff/Turner (1989), Weber (1995), Kövecses (1999). 
Erfahrungen sind damit verbunden, dass ein Ventil eine Art Schalter für Flüssigkeiten und gasförmige Stoffe ist. Wenn das Ventil geschlossen ist, blockiert es den Durchstrom. Im metaphorischen Gebrauch des Lexems Ventil ist der neue, referenzielle Zustand (Zielbereich) durch den Bezug auf einen Ursprungsbereich kodiert. Im Satz Das Glücksspiel kann auch ein Ventil für Frustration sein. wird veranschaulicht, dass mit Hilfe des Ventils (= des Glücksspiels) ein Überdruck in diesem Stoff (= Frustration), in dem man etwas von dem Gas entweichen lässt, kontrolliert abgebaut werden kann. An dem Aufbau der Metaphern als sprachlichen Schöpfungen beteiligen sich die emotionalen Veranlagungen und alltägliche Beobachtungen.

\subsection{Ventil-Metapher: Beispiele}

Ich präsentiere die gesammelten Ventil-Metaphern stets in längeren Kontexten. Metaphern sollen nicht isoliert betrachtet werden, sondern in Bezug auf den gesamten Text, weil sie erst durch bestimmte Texteigentümlichkeiten ihre spezifische Bedeutung erfahren. Die Interpretationsrichtung entsteht jeweils durch rezeptionsorientierte Perspektive. Fettmarkierung verweist auf das Lexem Ventil und andere Textelemente, die metaphorische Bedeutung der Ventil-Ausdrücke implizieren; Unterstreichung - auf weitere Textelemente (falls vorhanden), die mit der Verwendung der Metapher korrelieren. Demnächst wird der gegebene Gebrauch der Metapher interpretiert.

Beispiel 1: $\mathrm{X}_{\text {[Nominalphrase] }}$ als Ventil benutzen

[...] Selbst wenn die Öffnung der Mauer vom Politbüro als Ventil benutzt wurde, dann war es ein Sieg der Massen und ihrer Forderung nach Freiheit jetzt. Die Öffnung ist gewiß für den organisatorischen Zeitplan der Opposition zu früh, aber andererseits - um Biermann zu zitieren - kommt Freiheit nie verfrüht (taz 16.11.89, S. 8).

Die Verbindung zwischen den Subjekten, herausgegriffen durch die Metapher (die Öffnung der Mauer $\rightarrow$ das Ventil), schafft eine Ähnlichkeit zwischen den Dingen, die zuvor und außerhalb des Kontextes zwischen denselben nicht gegeben war. Dieses Bemerken von Ähnlichkeiten ist damit weder Ursache noch Grund für die Metapher, sondern die Metapher ist das Resultat des Verstehens der jeweiligen Analogie, an die bisher wohl niemand gedacht hatte, d. i. einer neuen Sichtweise auf die aktuelle Situation (Ventil/Mauer öffnen $\rightarrow$ Blockade lösen). Der Sprecher imaginiert eine mögliche Situation aus seiner Erfahrung und erzeugt eine kontextuelle Bedeutung, die zugleich die Forderungen ( $\rightarrow$ nach Freiheit), Stimmungen $(\rightarrow$ Skepsis: selbst wenn ...) und Einstellungen zum thematisierten Sachverhalt der politisch-gesellschaftlichen Wirklichkeit $\rightarrow$ Sieg der Massen, gewiss zu früh, nie verfrüht) vermittelt. 
Beispiel 2: $\mathrm{X}_{[\text {Nomen] }}$ als Ventil + Genitivattribut instrumentalisieren

Unverbindlicher Massendialog oder Runder Tisch? [Überschrift] Die Partei versucht mit der neuen Offenheit aus der Defensive zu kommen, scheint aber ansonsten den Dialog als unverbindliches gesamtgesellschaftliches ,, brainstorming" zu betrachten. Die Partei hofft, den Dialog als Ventil der gesellschaftlichen Unruhe instrumentalisieren zu können. Für die Opposition geht es in erster Linie um Mobilisierung und Verbreiterung ihrer Basis und um die Überführung der Diskussion in gesellschaftlich autorisierte Verhandlungsrunden (taz 27.10.89, S. 6).

Das Lexem Ventil als das metaphorische Gegenstück zum Lexem Dialog wird durch das wörtlich zu verstehende Genitivattribut erweitert. Die Metapher konkretisiert die Gefahr, die ähnlich wie durch falsches Benutzen des Geräts verursacht werden kann. Dies bedeutet nicht, dass man sich die Metapher als Bild vorstellt, sondern ihre Wirkung auf die Erzeugung einer gefühlsmäßigen Einstellung abzielt. Der situative Kontext stellt sich wie folgt dar: Die negative Stellungnahme der Opposition richtet sich gegen die SED, die laut den Vertretern der Opposition mit dem Versuch, sich an die Spitze der Bewegung zu stellen, die Bürgerbewegung kanalisieren möchte, und zwar durch zahllose Dialogangebote.

Beispiel 3: ein Ventil schaffen (zum Zweck von etwas)

Die auffällige Dichotomie der Erklärung [...] offenbarte zwar zum ersten Mal Interessendivergenzen auch innerhalb der höheren Führungsebene der Partei. Doch in ihrer strategischen Intention werden sich beide Seiten einig gewesen sein: Ein Ventil zu schaffen, um zu beschwichtigen und die Diskussion auf jenem Terrain zu führen, wo die SED Heimvorteil genießt. Der Marschplan sah vor, wankelmütige Genossen noch einmal auf Linie zu bringen, der Kirche als einzig legitimer Organisation ein Gesprächsangebot zu unterbreiten, um so die Händel innerhalb der Opposition anzufachen und nicht zuletzt damit die Exponenten der Opposition weiter im gesellschaftlichen Abseits zu halten (taz 18.10.89, S. 8).

Dem metaphorischen Ausdruck wird die wörtlich zu verstehende Finalangabe angeschlossen. Daraus ergibt sich eine gewisse Zweiteilung, nämlich in einen metaphorischen Fokus (der nominale Kern des metaphorischen Ausdrucks), der für das steht, worüber die Metapher etwas aussagt ( $\rightarrow$ Interessendivergenzen, die auffällige Dichotomie), und die übrigen, nicht metaphorischen Teile des Satzes, die die Funktion haben, auszudrücken, was über die Metapher ausgesagt wird $(\rightarrow$ Zweck, zu dem „ein Ventil geschaffen wird“). Die Metapher fungiert als Filter, mittels dessen die Leser ihre Ansichten über den Hauptgegenstand (re)organisieren können. 
Beispiel 4: $\mathrm{X}_{[\text {Nomen] }}$ der Ventile [Genitivattribut, Plural]

Die Politik der Ventile hat die Akutheit der Krise nur verschoben. Ab jetzt wird entschieden werden müssen, ob die Antwort Repression ist oder Reform (taz 7.10.89, S. 8).

Der attributive Gebrauch gibt den komplexen Sachverhalt der politischen Wirklichkeit komprimiert wieder, ohne ihn detailliert beschreiben bzw. erläutern zu müssen (Die Grundannahmelautet: Das alltägliche Wissen über das Funktionieren des Ventils ist vorhanden). Die Metapher übernimmt an dieser Stelle eine zusammenfassende und sprachökonomische Funktion, hat aber auch eine konkrete Wertungsrichtung. Sie ist ein subjektiver Kommentar des Verfassers zu politischen Entscheidungen (die Krise ist $\rightarrow$ nur verschoben, nicht vermieden), die er übrigens an anderer Stelle seines Textes auch als die Politik des Rettet-das-Jubiläum bezeichnet.

Beispiel 5: [[Nom]-Ventil $]_{\text {Kompositum }}$ öffnen

Wahrscheinlich wollte Krenz mit diesem Zug eher das Sicherheitsventil öffnen als einen Schritt zur deutschen Einheit zu tun. [...] Vielleicht sei der Tag nicht mehr fern, an dem die vier Alliierten einen Friedensvertrag mit Deutschland unterzeichnen. [...] Der Prozeß einer europäischen Annäherung sei nun beschleunigt worden (taz 13.11.89, S. 10).

Es liegt eine Übertragung von einem (alläglichen, technischen) Anwendungsbereich des Ausdrucks in einen anderen (politischen) vor, wobei die einzelnen Vorstellungskomplexe zu diesem Ausdruck sich nur in ihrer Spezifik des jeweiligen Anwendungsbereichs unterscheiden. Mentale Prozesse, die beim Öffnen des Sicherheitsventils entstehen, bilden die Grundlage für das Sinnverstehen des neuen Kontextes. In dieser Hinsicht erfolgen die Sichtweisen über denselben (VentilÖffnen-)Prozess, der in einem neuen Kontext, aus dem die Metaphorisierung hervorgeht, letztendlich als metaphorisch zu charakterisieren ist (Zug $\rightarrow$ Ventil). Die Formulierung rekurriert auf Einstellung des Schreibenden zu der damals neuen, etwas weniger restriktiven Visapolitik Deutschlands; sprachlich ist sie sehr vorsichtig formuliert: wahrscheinlich, vielleicht, eher, indirekte Rede.

Beispiel 6: [ [adj. Attribut] Ventil-[Nom] $]_{\text {Kompositum }}$ spielen

Der Verlust der gesellschaftlichen Kontrolle, mit den Demonstrationen der letzten Wochen schon offenkundig, wird mit der Öffnung der Massenmedien weiter vorangetrieben. Das gilt auch dann, wenn die partielle Liberalisierung im Kalkül der Partei nur eine von oben gesteuerte Ventilrolle spielen 
sollte. Sie tangiert die Struktur kontrollierter Öffentlichkeit und damit eine der wesentlichen Stützpfeiler des alten Systems (taz 21.10.89, S. 8).

Im öffentlichen politischen Diskurs des Jahres 1989 (Beispiele 1-7) wird die VentilMetapher immer wieder bei Themen der Öffnung: der Massenmedien, der Mauer, der Grenzen etc. verwendet, und zwar über die Vorstellung von Aufgaben der Ventile, übertragen auf die abstrakten Elemente (Mechanismen) der politischen Wirklichkeit. Die metaphorische Sichtweise auf die politische Situation kreiert letztendlich den stilistisch markierten Sprachgebrauch des Verfassers. Und die Metapher selbst ist die sprachliche Manifestation seiner Einstellungen. Im Beispiel 6 signalisiert sie kritische Überzeugung des Autors über die Strategie der Partei, bei der andere nur zu Marionetten im Spiel werden, mitgesteuert durch Attribute zum nominalen Kopf des Ausdrucks (nur, eine von oben gesteuerte zu Ventilrolle).

Beispiel 7: [Ventil-[Nom] $]_{\text {Kompositum }}$ haben

Mit deutlicher Skepsis bis vorsichtigem Optimismus reagiert die DDR-Opposition auf die zurückhaltenden Reformsignale aus der Führungsetage der Partei. Die Erklärung der Partei habe offensichtlich Ventilfunktion (taz 13.10.89, S. 3).

Semantisch hat der Ausdruck den Status einer okkasionellen Wortverbindung, wobei Ventil die metaphorische Komponente bildet, während Funktion haben in freier Bedeutung verwendet wird. Das Kompositum Ventilfunktion muss seinerseits wörtlich und metaphorisch verstanden werden, wenn man den Bereich dessen erfassen will, was durch die ganze Wortverbindung abgedeckt wird. Im metaphorischen Sinn signalisiert die Formulierung Ventilfunktion haben einfach die Stellungnahme des Verfassers; zusätzlich durch das Modaladverb offensichtlich mit einem denkbar hohen Wahrscheinlichkeitsgrad kommentiert.

Beispiel 8: ein Ventil für die Wut ${ }_{\text {Emotionswort] }}$ (sein) [lexikalisierte Metapher] Beispiel 9: der Wut ${ }_{[\text {Emotionswort] }}$ ein Ventil verleihen

Ein Ventil für die Wut (Überschrift; Focus 10.10.2011 10 ). Der Artikel thematisiert Massenstreiks in Griechenland in Folge der wirtschaftlichen Vorgaben der Troika (EU, IWF, EZB), die völlig entgleiste griechische Wirtschaft durch Reformen innerhalb von vier Jahren wieder aufrichten will. Im weiteren Text: [...] Aber die Streiks verschlimmern doch nur alles. Sie sind unser

10 Online Ausgabe: FOCUS Magazin | Nr. 41 (2011) Montag 10.10.2011, https://www.focus.de/ politik/deutschland/politik-ein-ventil-fuer-die-wut_aid_672968.html. Zugriff am 26.10.2018. 
einziges Gegenmittel. Damit bringen wir unsere Wut und Verzweiflung zum Ausdruck. Es geht nicht um die Arbeitsniederlegung als solche. Sie ermöglicht, Versammlungen abzuhalten, zu diskutieren und der Wut ein Ventil zu verleihen. Anders kann man die Menschen nicht mehr ruhig halten. Sonst hätten wir hier noch mehr Chaos und Verzweiflungstaten. Sie werden zurzeit keinen Griechen finden, der ruhig, verständnisvoll und gelassen über seine Lage reden kann. Die Menschen wissen nicht, was ihnen am nächsten Tag blüht. Wenn wir still bleiben, meinen die Regierenden, alles wäre gut so. Es ist aber überhaupt nichts mehr gut!

Die beiden Beispiele (und auch 10,11) unterscheiden sich von den bisherigen dadurch, dass Emotionen nunmehr direkt benannt werden. Die gebräuchliche VentilWut-Metapher erscheint in 8, 9 im Bezugsbereich der Streik/die Streiks in Griechenland; sie wird durch die Interaktion mit den Formulierungen, die physiologisch-psychische Reaktionen der Menschen im Bereich des Emotionalen benennen (Wut und Verzweiflung), als emotionsbezeichnend unterstützt.

Beispiel 10 und 11: ein Ventil suchen

Wende-Generation ,Die Frustration sucht ein Ventil“ (Überschrift; Frankfurter Rundschau, 14.10.2017 ${ }^{11}$ ). Die Überschrift ist die Aussage des Psychologen Jörg Frommer über psychische Leiden von Ostdeutschen und ihre politischen Konsequenzen.

Hier sucht jemand ein Ventil, um seinem/ihrem Ärger Luft zu machen und wählt dafür einen Raum, in dem schnelle Rückmeldungen, rasche Eskalation, lange (Wieder-)Abrufbarkeit und große Reichweite (ggf. haben auch der I die Schulpreisträger/in, die Jury, die Schulleitung Zugang zum Text) sehr wahrscheinlich sind. (Aus: Die demokratische Pflicht und das Sprachsystem. Erneute Diskussion um einen geschlechtergerechten Sprachgebrauch von G. Zifonun, in: IDS Sprachreport 4/2018:49).

Emotionswörter in diesen Beispielen nehmen verschiedene Satzpositionen ein: Frustration steht an Subjektstelle (10); über Emotion des Subjekts (Ärger) wird in angeschlossener finaler Konstruktion gesprochen (11). Frustration und Ärger halten sich im gleichen semantischen Rahmen wie die (negative) Emotion Wut (Beispiele 8, 9). Der prototypische Assoziationskomplex zu Ventil im allgemeinen Verstehen des Ventils als einer Art regulatives Gerät (das Ventil ist Vorrichtung, mit der das Auslassen von Flüssigkeiten oder Gasen gesteuert wird) wird in einen neuen Bereich des Alltagslebens übertragen. Das bedeutet folglich, dass im me-

11 Online Ausgabe: http://www.fr.de/politik/wende-generation-die-frustration-sucht-ein-ventil-a-136 8025. Zugriff am 26.10.2018. 
taphorischen Sinn Emotionen (Frustration, Ärger, Wut, Verzweiflung) eben diese Flüssigkeiten in einem Behälter sind. Intensivierung (wie dies z. B. beim Kochen der Fall ist) lässt die Temperatur der Flüssigkeit steigen und der Dampf sucht den Auslassweg aus dem Behälter. Über diese Assoziierung sucht man eine entsprechende Darstellungsweise für die Intensivierung der Gefühle.

Beispiel 12: ein Ventil aufmachen

„Polen hat ein Ventil aufgemacht" (Überschrift) Luxemburgs Außenminister Asselborn über die Kaczynskis und die EU-Reform (aus einem Interview) [...] der Brüsseler EU-Gipfel im Juni wäre im Streit um die künftige Machtverteilung in Europa fast an den Forderungen des polnischen Führungsduos Lech und Jaroslaw Kaczynski gescheitert. Vor dem Gipfel hatte Polens Regierungschef Jaroslaw Kaczynski im Streit um das Stimmengewicht sogar die polnischen Weltkriegstoten ins Spiel gebracht. [...] Das war etwas, was -aus polnischer Sicht - einmal heraus musste. Das polnische Führungsduo hat da ein Ventil aufgemacht. [...] (Der Tagesspiegel, 17.07.2007'12)

Die metaphorische Formulierung $X$ hat ein Ventil aufgemacht passt in das allgemeine Interpretationsmodell der Ventil-Metapher (Beispiele 8-11): „den Emotionen Luft machen, Emotionen abreagieren“. Sie werden metaphorisch „kondensiert"; wenn die Stimmung eskaliert, müssen sie - ähnlich wie das Fluidum bei hohen Temperaturen aus dem Behälter - heraus.

Beispiel 13: $\mathrm{X}_{[\text {Nomen] }}$ ohne Ventil

Beispiel 14: Ventile $[$ Plural] finden

Beispiel 15: Ventile $[$ Plural] verstopfen

Kritik ohne Ventil (Überschrift) »TV-Duell«: Die großen Parteien sind vor aller Augen zum Block verschmolzen. Kommentar nach einer TV-Gesprächsrunde zwischen Angela Merkel und Martin Schulz: ,[...] 30 Prozent der Deutschen hätten sich bereits komplett abgewendet von den etablierten Parteien, wählten entweder AfD oder bewusst gar nicht mehr, hieß es. Die seien auch nicht mehr zurückzuholen, prognostizierte eine Journalistin des „Spiegel", ohne dafür Widerspruch zu ernten. [...] Das eher gelangweilt als erzürnt aufgenommene „Platzpatronen-Duell“ geriet zum perfekten Abbild der deutschen Misere. [...] Der Spruch „Die sind doch eh alle gleich “ erhielt seine Bestätigung von höchster Stelle. [...] In der Geschichte entpuppten sich

$12 \mathrm{https} / / /$ www.tagesspiegel.de/politik/polen-hat-ein-ventil-aufgemacht/988998.html. Zugriff am 26.10. 2018. 
derartige Phasen vordergründiger Friedhofsruhe oftmals als Vorstufe abrupter Umbrüche. Denn es sind Perioden der Gärung, in denen sich das explosive Gemisch der Unzufriedenheit sammelt, ohne bei der großen Politik Ventile zu finden, die es in einer lebendigen Demokratie haben sollte. Etablierte Politik und Medien haben die Ventile mit Verdikten wie „Populismus“, „Hetze“ oder „Spaltung der Gesellschaft“ derart fest verstopft, dass so gut wie nichts mehr entweichen kann. Sie halten diese Verstopfung für ihren Sieg. Das könnte sich als größter Irrtum der deutschen Geschichte seit den Fehleinschätzungen der SED am Ende der DDR herausstellen. "(von Hans Heckel) (Preußische Allgemeine Zeitung, Das Ostpreußenblatt, 06.09.2017 ${ }^{13}$ ).

Über die Aktualisierung übertragener Bedeutungen der drei Ausdrücke mit Ventil bekommt man auch den Bereich des Emotionalen in den Fokus. In (13) drängen naturhaft die negativen Emotionen, die nun als Kritik „heraus“ sind. Sie nehmen sogleich einen Substanzcharakter an und sind als Unzufriedenheit (das explosive Gemisch der Unzufriedenheit) versprachlicht, wobei Ventil (in 14) als regulative Vorrichtung (metaphorisch für Unterdrückung von Emotionen) in Frage kommt. In (15) liegt eine attributive und modal-konsekutive Konkretisierung vor: Ventile mit Verdikten wie „Populismus “, „Hetze“, „,Spaltung der Gesellschaft" derart fest verstopfen, dass so gut wie nichts mehr entweichen kann.

Beispiel 16: ein Ventil finden

Proteste. Spaniens Mutbürger finden ein Ventil (Überschrift). In Madrid protestieren seit Tagen Tausende gegen Politik und Banken. (Sie haben) [...] sich an der Puerta del Sol, dem zentralen Platz Madrids, unter improvisierten Zelten eingerichtet [...], um dort ihre Stimme für ,einen wirklichen politischen und sozialen Wandel" in Spanien zu erheben. (Von Martin Dahms), (stuttagrter-zeitung.de, 19.05.2011 14 ).

Analog zu früheren Beispielen wird die Intensivierung der Gefühle zur metaphorischen Interpretation beigesteuert (Proteste sind Wirklichkeitsgegenstand; das, worauf sich die Metapher bezieht), und zwar über die Vorstellung, dass Emotionen Flüssigkeiten in einem Behälter sind. Davon wird im Text nur ein bestimmter Anteil mitthematisiert: Ein Ventil wurde eingestellt (hier: gefunden) und daher können die Flüssigkeiten (= Emotionen) nun entsprechend aus dem Behälter herausfließen. Emotionen vermitteln als Auslösemechanismus zwischen Reiz und Reaktion. Aus

$13 \mathrm{https} / / /$ www.preussische-allgemeine.de/nachrichten/artikel/kritik-ohne-ventil.html.Zugriffam 26.10. 2018.

14 https://www.stuttgarter-zeitung.de/inhalt.proteste-spaniens-mutbuerger-finden-ein-ventil.3a1578e90844-4209-acab-d6550cad7f60.html. Zugriff am 26.10.2018. 
dem Gebrauchskontext der Metapher lassen sich Emotionen der Wut und der Unzufriedenheit mit der aktuellen Politik und der Politik der Banken einordnen.

\subsection{Ergebnisse}

Zur Struktur der Ausdrücke mit Ventil. In morphosyntaktischer Hinsicht geht das Lexem Ventil in folgende Strukturen einher:

- Nomen-Verb-Verbindungen, das Nomen steht in Sing. oder Plural: ein Ventil schaffen, (etw.) ein Ventil verleihen, ein Ventil suchen, ein Ventil aufmachen, Ventile finden, Ventile verstopfen, ein Ventil finden;

- Das Nomen Ventil geht als Bestimmungswort in die Nomen-Verb-Verbindung einher: eine Ventilrolle spielen, Ventilfunktion haben;

- Das Nomen Ventil ist Grundwort im nominalen Bestandteil der NomenVerb-Verbindung: das Sicherheitsventil öffnen;

- Vergleichsstrukturen: etwas als Ventil benutzen, etwas als Ventil instrumentalisieren;

- das Nomen hat die Funktion des Attributs; wird auch pluralisiert: die Politik der Ventile, Kritik ohne Ventil;

- das Nomen wird attribuiert: ein Ventil für die Wut, Ventile mit Verdikten wie „Populismus“, „Hetze“, ,Spaltung der Gesellschaft".

Zur Metaphorik. Eine Metapher entsteht durch ihre kontextuelle Verwendung. Das Substantiv Ventil ist in allen obigen Kontexten als metaphorisch zu charakterisieren. Jedoch kann die Metaphorizität in einigen Beispielen allein aus der sprachlichen Form der Ausdrücke gar nicht abgeleitet werden (vgl. ein Ventil finden, ein Ventil aufmachen, das Sicherheitsventil öffnen). Für meine Überlegungen war nun wichtig, in welcher lexikalischen Umgebung das Lexem Ventil in einem Kontext vorkommt. Schauen wir uns das Beispiel 16 an: Proteste. Spaniens Mutbürger finden ein Ventil. Der im Textverlauf geschilderte Grund für Proteste in Spanien ist Unzufriedenheit mit der aktuellen Politik und der Politik der Banken; es ist - wie dies die Metapherforschung formuliert - der Wirklichkeitsgegenstand; das, worauf sich die Metapher bezieht. Die Subjektstelle wird von Spaniens Mutbürger eingenommen, wobei das Wortspiel Mitbürger $\rightarrow$ Mutbürger den situationellen Referenzbezug ( $\rightarrow$ Proteste) garantiert. Erst durch Interaktion mit dem Kontext wird die metaphorische Lesart von ein Ventil finden aktualisiert. Die aktualisierte wörtliche, ursprüngliche Bedeutung des Ventils: Ventil ist eine Vorrichtung, mit der das Ein-, Durch- oder Auslassen von Flüssigkeiten oder Gasen gesteuert wird, wird auf den anderen Anwendungsbereich, die politische Situation und den Bereich des Emotionalen, übertragen. Es entsteht eine spezifische, mentale Repräsentation (Vorstellung) dadurch, dass eine verbindende Relation zwischen 
der alltäglichen Ventilvorstellung und der Vorstellung von diesen anderen (neuen) Sachverhalten gebildet wird. Rekapitulierend: Wenn man das Wort Ventil liest, hängt es von der lexikalischen Umgebung ab, welche der Bedeutungen realisiert wird. Um die Metapher als solche zu identifizieren und sie richtig zu verstehen, muss man den situativen Kontext, in dem sie verwendet wurde, mit berücksichtigen. Und umgekehrt, man muss auch den prototypischen Assoziationskomplex zum Wort kennen, damit die Kommunikation durch Metaphern im durch den Sprecher angenommenen Sinn erfolgt.

Neben den Ausdrücken, die daraus schöpfen, was man mit dem Ventil machen kann (... suchen, finden, aufmachen, verstopfen) und der gebräuchlichen emotionsbezeichnenden Ventil-für-Wut-Metapher, werden neue Wortverbindungen wie die Politik der Ventile, Kritik ohne Ventil gebildet. Neu sind sie nicht darum, dass sie zum ersten Mal gebildet wurden, was tatsächlich ja auch nicht nachvollziehbar ist. Die Wortverbindungen sind neu, weil sie nicht einem bereits üblichen Übertragungsweg entsprechen.

Zum Emotionalen. Deutsche Wörterbücher verzeichnen als lexikalisierte Metapher bei Ventil den Ausdruck, den die Emotion Wut benennt ${ }^{15}$, im Belegkorpus vgl. Beispiele 8, 9. Nun ist aber zu beobachten, dass nach dem gleichen Interpretationsmodell Ausdrücke gebildet werden, die auch andere Emotionen benennen und darüber hinaus soziale Eigenschaften bzw. Verhaltensweisen bezeichnen können. Die Schreibenden bedienen sich der Analogie dazu, wie ein Ventil funktioniert, um sinnvolle Erläuterungen der neuen Sachverhalte (auf die hin projiziert wird) zu erzeugen, Markierungen zu vermitteln, Bewertungen zu äuBern, Argumentationen zu stützen etc. Diese anderen Emotionen halten sich in einem semantischen Rahmen der Wut; es sind Ärger, Frustration, Unzufriedenheit. Über Verhaltensweise und Eigenschaften sprechen okkasionelle Verbindungen wie die Politik der Ventile, Kritik ohne Ventil. Alle Ausdrücke mit Ventil, die in untersuchten Textabschnitten auf verschiedene Situationen verweisen, können als eine Äußerung der subjektiven Stellungnahme ihrer Autoren interpretiert werden, die jeweils negative Einstellungen oder Bewertungen signalisiert.

Die Durchsicht der Ausdrücke mit Ventil mag eine Assoziation in Hinblick auf Konzeptualisierung und Benennung von Emotionen verschiedener Art geweckt worden sein, wovon die bekannteste Konzeptualisierung die BehälterMetapher ist (vgl. u. a. Lakoff/Johnson 1980, in weiteren Untersuchungen z. B. von Kövecses 1999, Schwarz-Friesel 2007). Die zugrundeliegende Konzeptualisierung lautet: Emotionen sind Flüssigkeiten in einem Behälter. Die Vorstellung des Aufmachens und Zumachens des Ventils bei einem Behälter entspricht diesem

15 Schwarz-Friesel (2007:199) äußert eine wichtige Bemerkung: Metaphern können emotionsbezeichnend oder emotionsausdrückend sein. Metaphern in meinem Belegkorpus sind entweder emotionsbezeichnend (man benannte Wut, Frustration, Verzweiflung, Ärger, Unzufriedenheit) oder emotional neutral. Die ,neutralen“ eigneten sich aber gut für die Vermittlung von Stimmungen, Einstellungen, Bewertung. 
allgemeinen Bild folgendermaßen: Bei der Intensivierung der Gefühle werden sie mit Hilfe des Ventils aus dem Behälter ausgelassen, bei der Maskierung und Unterdrückung von Gefühlen werden sie im Behälter verschlossen, mit dem Ventil verstopft. Die entsprechenden sprachlichen Realisierungen dieses konzeptuellen Typs sind unter meinen Beispielen: Die Frustration sucht ein Ventil, Kritik ohne Ventil, Hier sucht jemand ein Ventil, um seinem / ihrem Ärger Luft zu machen, Ein Ventil für die Wut. In anderen Beispielen, wenn über Emotionen nicht direkt referiert wird, wird die Haupteigenschaft des Ventils als Regulator einer zu bestimmenden Druckkraft, als Druck-Regler akzentuiert (das Ventil öffnen, das Ventil aufmachen, das Ventil ist verstopft). Sie benennen zwar keine Emotionen, aber tragen allgemein wesentlich zur Reflexion bei, da sie besonders effektiv dazu verwendet wurden, Einstellungen, Urteile und Markierungen zu vermitteln und entsprechende (möglicherweise auch emotionale) Einstellungen der Leser zu steuern.

Zur Herstellung der Textkohärenz. Die metaphorischen Ausdrücke beteiligen sich an dem Prozess der Herstellung von Textkohärenz, indem sie als Referenzpunkte verschiedene Textstellen innerhalb des Textes verknüpfen. Daraus erfolgt ihre textstrukturierende Funktion. Die Ventil-Metapher nimmt die folgenden Positionen ein: Die initiale und zugleich interpretationsanleitende Position in Schlagzeilen, Überschriften, Unterüberschriften und Anfangsabschnitten (Beispiel 13), die Endposition mit der reaktiven oder zusammenfassenden Funktion (Beispiel 7) und eine rahmenbildende Position, d. h. die Metapher wird als Interpretationsanleitung eingesetzt und die Rezeptionsrichtung wird mit gleicher Metapher zum Schluss bestätigt (Beispiel 12). Im Textverlauf wird das metaphorische Ventil durch den Textinhalt, oft attribuiert, elaboriert; die metaphorische Formulierung wird dadurch zur Veranschaulichung der abstrakten Sachverhalte benutzt und um der Argumentation Nachdruck zu verleihen (Beispiele 14, 15).

\section{Schlussbemerkungen}

Das Wesen der Ventil-Metapher besteht darin, dass konkrete Erfahrungen auf eine spezifische Prototypik hin zusammengefasst werden. Dadurch wird ein Verstehen von neuen Sachen und Sachverhalten, die in ihren konkreten Erscheinungsformen jedoch mehr oder weniger von dem Prototyp abweichen können, ermöglicht. Mit anderen Worten: Im Prozess der Metaphorisierung, ich würde auch sagen - im Prozess der spezifischen Sichtweise des „Sehen-als“ - werden Beziehungen zwischen einem standardisierten Sinnverständnis des Ventils als regulativem Gegenstand und konkreten, empirischen Vorgängen geschaffen. Die Annahmen stellen sich wie folgt dar: Wenn immer wir etwas nicht verstehen, können wir zu unseren (Alltags- und Umwelt-)Erfahrungen greifen $(\rightarrow$ Ventil als Gegenstand des Alltags). So lässt sich etwas einordnen, das sich theoretisch nicht einfach er- 
klären lässt ( $\rightarrow$ Projektion auf den neuen, politischen Bereich). Wenn uns etwas verunsichert, können wir über den Vergleich mit dem Bekannten, Vertrauten und Fassbaren es wenigstens annährend richtig erfassen bzw. verständlicher machen ( $\rightarrow$ eine Analogie zwischen den beteiligten Referenten wird behauptet).

Ein Schlusswort zum interpretativen Charakter der Analyse. Die Suche nach metaphorischen Strukturen ist zum Instrument meiner Analyse geworden. Im Laufe dieser, wenn auch wegen geringer Anzahl der Beispiele unvollständigen Analyse, konnte unterschieden werden, was an der Themaentfaltung metaphorisch ist. Die diskutierten Metaphern, jedoch auch andere vorherrschende wie neu aufkommende Metaphern, weisen explizit darauf, dass der Metapher nicht als einem ausschließlich linguistischen (an der sprachlichen Form gebundenen) Phänomen beizukommen ist, und die Erklärungsversuche auf kognitive Unterstützung, d. i. auf eine kognitive Grundlage angewiesen sind. Wenn man wie in Rahmen dieser Diskussion annehmen soll, dass die Metaphern aus Ausdrucksnot angesichts des schwer Unfassbaren, somit auch der oft so empfundenen Unfassbarkeit und Unaussprechlichkeit des Gefühls entstehen, mag nicht wundern, dass durch Metapher die Vorstellung (möglicherweise die Illusion) entsteht, dass auch Emotionen kognitiv durchdringbar sind. Wesentlich ist hier, dass Zuschreibung von Emotionen, von welchen sich der Schreibende tatsächlich leiten lässt, auf Interpretationen beruht. Ebenso interpretativ ist, welche (andere oder ähnliche) Gefühle die verwendeten Metaphern beim Leser stimulieren können; „Flüssigkeit im Behälter" ist wohl ambivalent. Die Einordnung von Emotionen hängt in erster Linie von unserer Lebenserfahrung, unserem Weltwissen, wohl auch der Intuition, Eintragungen in unser mentales Lexikon, kognitiven Schemata ab. Es handelt sich bei Assoziationskomplexen (assoziierten Implikationen) zu jeweiligen neuen Zusammenhängen um instabile Größen. Demzufolge sind auch immer Interpretationsalternativen gegeben.

\section{Literatur}

Abdulrahman Abdulhamid, 2018, Tiermetaphorik in unterschiedlichen Diskurstraditionen, Berlin u. a.

BŁAchut Edyta, 2014, Bewerten - Semantische und pragmatische Aspekte einer Sprachhandlung, Hamburg.

Bowers John Waite / MetTs Sandra M. / Duncanson Thomas, 1985, Emotion and interpersonal communication, in: Knapp M.L./Miller G.R. (Hrsg.), Handbook of interpersonal communication, Beverly Hills, S. 502-559.

Cirko Lesław, 2019, Emotionen in akademischen Gutachten, in: Studia Linguistica XXXVIII, S. 7-20.

Duden. Deutsches Universalwörterbuch. Das umfassende Bedeutungswörterbuch der deutschen Gegenwartssprache, 2011, 7. Aufl., Mannheim.

EBert Lisanne / Gruber Carola / Meisnitzer Benjamin / RetTinger Sabine (Hrsg.), 2011, Emotional Grenzgänge. Konzeptualisierungen von Liebe, Trauer und Angst in Sprache und Literatur, Würzburg. 
ELSEN Hilke, 2014, Linguistische Theorien, Tübingen.

FieHLER Reinhard, 1990, Kommunikation und Emotion. Theoretische und empirische Untersuchungen zur Rolle von Emotionen, Berlin.

FieHLeR Reinhard, 2002, How to Do Emotions Withs Words: Emotionality in Conversations, in: Fussell S.R. (ed.), The verbal communication of emotion. Interdisciplinary perspectives, Mahwah u. a., S. 79-106.

FreITAG Rainer, 1975, Zum Verhältnis von Expressivität, Gefühlswert und Wertungskomponente im aktuellen Sprachgebrauch, in: LS/ZISW/A, Heft 14, Berlin, S. 147-155.

FrIELING Gudrun, 1996, Untersuchungen zur Theorie der Metapher. Das Metaphern-Verstehen als sprachlich-kognitiver Verarbeitungsprozeß, Osnabrück.

FrIES Norbert, 2000, Sprache und Emotionen: Ausführungen zum besseren Verständnis; Anregungen zum Nachdenken, Bergisch Gladbach.

Fries Norbert, 2004, Gefühle, Emotionen, Angst, Furcht, Wut und Zorn, in: Börner W. (Hrsg.), Emotion und Kognition im Fremdsprachenunterricht. Tübingen, S. 3-24.

Hermanns Frank, 2002, Dimension der Bedeutung: Aspekte der Emotion, in: Cruse D.A. et al. (Hrsg.), Lexikologie/Lexicology, HSK, Bd. 1, Berlin u. a., S. 356-362.

KirchHoff Susanne, 2015, Krieg mit Metaphern. Mediendiskurse über 9/11 und den „War on Terror", Berlin/New York.

Kittay Eva Feder, 1987, Metaphor: Its Cognitive Force and Linguistic Structure, Oxford.

KöveCSES Zoltán, 1990, Emotion concepts, New York u. a.

KöVECSES Zoltán, 1998, Are there any emotion-specific metaphors?, in: Athanasiadou A./Tabakowska E. (Hrsg.), Speaking of emotions. Conceptualisation and expression, Berlin u. a., S. $127-151$.

KövecSEs Zoltán, 1999, Metaphor and Emotion. Language, Culture and Body in Human Feeling, Cambridge.

LaKofF George / Johnson Mark, 1980, Metaphors we live by, Chicago.

LaKOFF George / JoHnson Mark, 2004, Leben in Metaphern. Konstruktion und Gebrauch von Sprachbildern, 4. Aufl., Heidelberg.

LAKOFF George / Turner Mark, 1989, More than Cool Reason. A Field Guide to Poetic Metaphor, Chicago.

Ludwig Klaus-Dieter, 1974, Zu einigen Fragen des Verhältnisses von Sprache, Wertung und Emotion, in: Sprachpflege 23, S. 51-54.

OrTnER Heike, 2014, Text und Emotion. Theorie, Methode und Anwendungsbeispiele emotionslinguistischer Textanalyse, Tübingen.

Pielenz Michael, 1993, Argumentation und Metapher, Tübingen.

SCHWARZ-Friesel Monika, 2007, Sprache und Emotion, Tübingen.

Skirl Helge / Schwarz-Friesel Monika, 2007, Metapher. Kurze Einführungen in die germanistische Linguistik 4, Heidelberg.

Weber Frauke, 1995, Denken in Metaphern: Kognitive Semantik und französische Gefühlsmetaphorik, Frankfurt a. M.

\section{Emotionalisation of Speaking and Metaphors: Metaphorical Expressions with Valve}

The present paper deals with the relationship between language, emotion and metaphor, theoretically and based on sets of examples. Firstly, I am going to discuss how emotions shape the language we use (based on the works of Fiehler 1990, Schwarz-Friesel 2007, Ortner 2014 and Ebert/Gruber/ 
Meisnitzer/Rettinger (eds.) 2011) and what the role of metaphors in descriptions of emotions is (based on the theory of Lakoff and Johnson 1980, Kövecses 1999). Secondly, using examples from the press I am going to show how the emotions of i.a. rage, irritation or frustration can be made verbal in a metaphorical sense. For this purpose, German metaphorical expressions with the noun Ventil 'valve' will be examined. I am using a descriptive-interpretive approach in my analysis.

Keywords: emotion, language, metaphor, metaphorical expressions with valve. 